\title{
Atypical complication in acute promyelocytic leukemia
}

\author{
Minodora Onisai ${ }^{1,2}$, Ana-Maria Vladareanu ${ }^{1,2}$, Iuliana Iordan ${ }^{1,2}$, Andreea Spinu1,2, \\ Ana-Maria Neagu ${ }^{1,2}$, Adrian Alexandru ${ }^{1,3}$ \\ ${ }^{1}$ „Carol Davila“ University of Medicine and Pharmacy, Bucharest, Romania \\ ${ }^{2}$ Hematology Department, Emergency University Hospital, Bucharest, Romania \\ ${ }^{3}$ Plastic and Reconstructive Surgery Clinic, Emergency University Hospital, Bucharest, Romania
}

\begin{abstract}
Acute promyelocytic leukemia (APL) is a distinct subtype of acute myeloid leukemia (AML) with a generally good prognosis but characterized by potentially severe hemorrhagic complications due to coagulopathy, and thus possibly high early mortality. We present the case of a young patient with APL, with a complication which was unresponsive to conservative treatment and required surgical resection, namely herpetic lesion complicated with infection and necrosis. This case sustains the importance of teamwork in hematologic patients.
\end{abstract}

Keywords: acute promyelocytic leukemia, coagulopathy, infections, surgical resection

\section{INTRODUCTION}

Acute promyelocytic leukemia (APL) is a distinct subtype of acute myeloid leukemia (AML) characterized by potentially severe hemorrhagic complications due to coagulopathy (1). It accounts for approximately $5-20 \%$ of AML cases (2). In APL patients requiring surgery, the risks and benefits must be carefully evaluated, especially at diagnosis, as coagulopathy and thrombocytopenia may increase the risk of fatal bleedings.

We present the case of a young patient with APL, with an infectious complication with indication for surgical resection, namely herpetic lesion complicated with infection and necrosis.

\section{CASE PRESENTATION}

A young female patient, 34 years old, with no relevant medical history, presented to the Emergency Department for persistent menorrhagia for about a month and weight loss of approximately 5 $\mathrm{kg}$ in a few weeks. She had no exposure to a toxic environment, she was not a smoker, and she had had three normal pregnancies. She had no brothers or sisters and no familial medical history.

The clinical examination was unremarkable except for pallor and confirmed genital bleeding, without other active hemorrhages; ECOG 0. The pregnancy test was negative.

Laboratory examination revealed pancytopenia: moderate normocytic anemia - hemoglobin (HGB) $9.5 \mathrm{~g} / \mathrm{dl}$, medium cell volume $89 \mathrm{fL}$, mild leucopenia - white blood cell count (WBC) 3,700/ $\mathrm{mm}^{3}$, but severe thrombocytopenia - thrombocyte

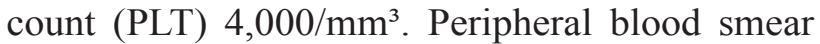
showed atypical promyelocytes $44 \%$, neutrophils $21 \%$, eosinophils $2 \%$, basophils $2 \%$, lymphocytes $25 \%$, and monocytes $6 \%$. Erythrocyte sedimentation rate and LDH level were increased, with values of $40 \mathrm{~mm} / 1 \mathrm{~h}$, respectively $250 \mathrm{U} / 1$. Viral markers were negative for hepatitis $\mathrm{B}$ and $\mathrm{C}$, and for HIV infection.

We also observed a decreased fibrinogen level, $120 \mathrm{mg} / \mathrm{dl}$, with normal coagulation times.

A bone marrow aspiration was performed, which showed $75 \%$ atypical promyelocytes: large cells, with round or incised nucleus, nucleoli pres- 
ent, slightly basophilic cytoplasm, with multiple granules and Auer rods (Figure 1A). They expressed myeloperoxidase highly (Figure 1B). Flow cytometry of bone marrow aspirate showed the presence of $64 \%$ precursor cells CD45+ CD33+ CD34- CD9+ CD10- CD3- CD7- CD2- CD56CD11b+/-CD117+ CD38+ HLA-DR-.

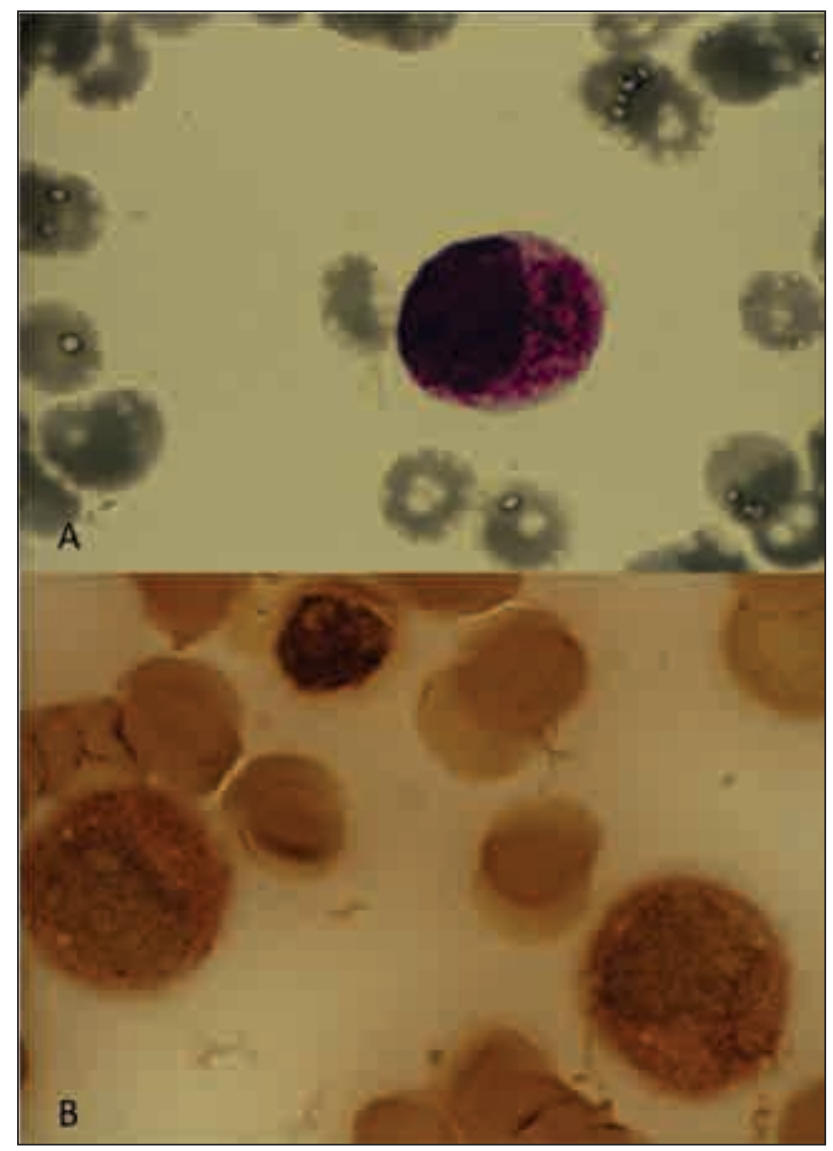

FIGURE 1. Microscopic images - bone marrow. 1A. Atypical promyelocyte. May-Grunwald-Giemsa stain 100X. 1B. Promyelocytes positive for myeloperoxidase. Myeloperoxidase stain 100X

Cytogenetic examination revealed the presence of $\mathrm{t}(15 ; 17)(\mathrm{q} 22 ; \mathrm{q} 21)$ translocation, and it was confirmed by fluorescence in situ hybridization. Molecular biology tests were positive for PML-RAR $\alpha$ and negative for FLT3-ITD and NPM1.

We thus established the positive diagnosis of APL with PML/RAR $\alpha$ fusion (according to World Health Organization 2016 criteria).

We started specific treatment, namely induction chemotherapy with AIDA protocol - all-trans retinoic acid (ATRA) $45 \mathrm{mg} / \mathrm{m}^{2} / \mathrm{d}$ and Idarubicin $12 \mathrm{mg} / \mathrm{m}^{2} /$ day on days $2,4,6,8$, together with supportive treatment: transfusions with platelets and packed red cells, hemostatic agents, allopurinol, herpes-zoster prophylaxis with Acyclovir $800 \mathrm{mg} /$ day and fungal infection prophylaxis with Posaconazole.
On the second day of treatment, the patient developed a small herpetic lesion on her lower lip, without any other symptoms. We supplemented antiviral medication with both local and systemic Acyclovir.

After ten days of treatment, full aplasia was installed: WBC $500 / \mathrm{mm}^{3}$ with $4 \%$ neutrophils, hemoglobin $6.2 \mathrm{~g} / \mathrm{dl}$, PLT $20,000 / \mathrm{mm}^{3}$; coagulation studies were normal. Infection and necrosis complicated the herpetic lesion (Figure 2), and the patient began to develop high fever. We started intravenous broad-spectrum antibiotics, including anti-anaerobe bacteria and anti-Staphylococcus; blood cultures were drawn, which were negative. The local evolution was not favorable due to the extension of the lesion, spontaneous bleeding, and persistent and aggravating fever. The patient maintained the severe aplasia despite transfusion support and specific treatment: WBC $1,500 / \mathrm{mm}^{3}$ with $30 \%$ neutrophils, HGB $6.7 \mathrm{~g} / \mathrm{dl}$, PLT 5,000/ $\mathrm{mm}^{3}$.

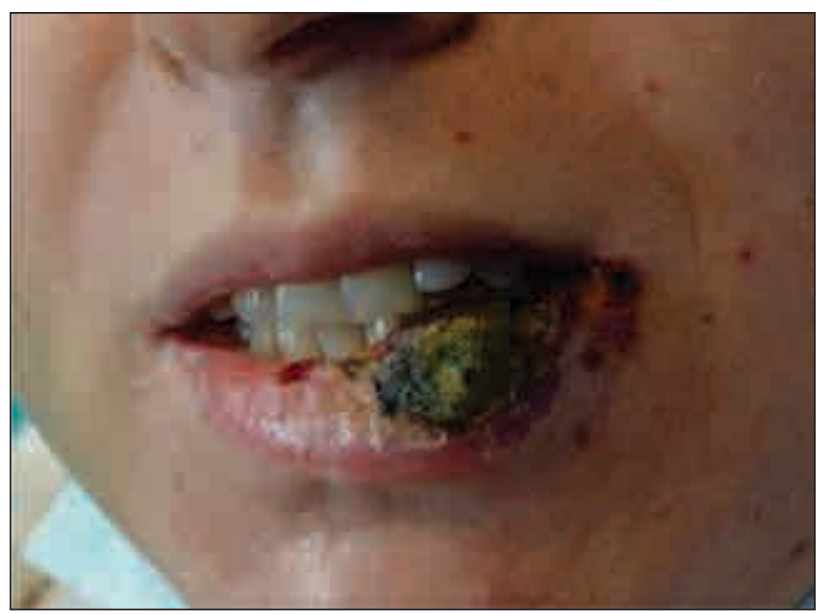

FIGURE 2. Herpetic lesion of the lower lip

We requested a plastic surgery consult. Because of the lack of response to conservative treatment, surgical intervention was recommended. The lesion resection was performed in a $\mathrm{V}$ shape, full-thickness excision with free margins. The lip defect was almost a third of the lip, which was closed primarily. Primary repair allowed us to reduce the duration of the surgery and risk of a potential bleeding and also provided a good outcome with minimal donor-site morbidity and the best color and texture match for the lip.

Thus, excision of the lesion was performed, with minimal bleeding during surgery, even though the patient presented severe thrombocytopenia. The evolution was rapidly favorable: no signs of inflammation or infection, minimal defect, and the fever resolved. Also, hematological features improved: WBC $2,000 / \mathrm{mm}^{3}$ with $50 \%$ neutrophils, HGB $10.5 \mathrm{~g} / \mathrm{dl}$, PLT 295,000/ $\mathrm{mm}^{3}$. Meanwhile, 
we continued the hematological treatment and the patient had semi-solid food for two weeks. Ten days after surgery, the lesion had a favorable evolution, without bleeding or signs of infection (Figure 3).
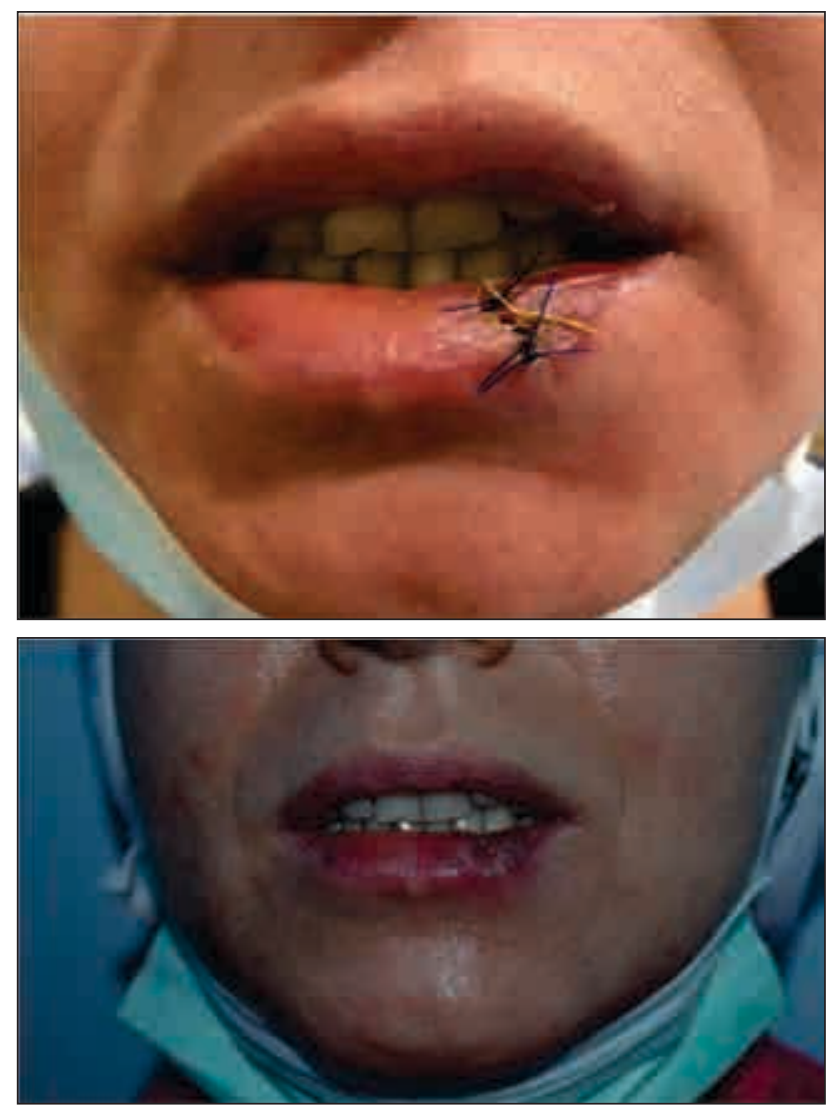

FIGURE 3. Herpetic lesion of the lower lip after ten days since surgical excision

Regarding hematological evaluation, the patient obtained complete hematological remission after induction therapy, with $2 \%$ (normal) promyelocytes in the bone marrow and no blasts. She continued with consolidation treatment for intermediate-risk acute promyelocytic leukemia (three courses in total, without any complications), and remission was stable - molecular examination for PML-RAR $\alpha$ was negative after induction and also after the three cycles of consolidation treatment. She has also finished maintenance treatment, preserving complete molecular remission.

\section{DISCUSSION}

At presentation, the most severe findings in our patient were thrombocytopenia and menorrhagia, which was thought to be secondary to thrombocytopenia. We excluded pregnancy and other frequent causes, which can associate thrombocytopenia $(3,4)$.

APL is a subtype of AML, which has particular morphologic and cytogenetic features, as well as specific treatment and better prognosis and survival compared to the other subtypes (1).

APL is characterized by a pathognomonic cytogenetic anomaly, namely the $t(15 ; 17)$ translocation, which translates into PML-RAR $\alpha$ mutation. Translocation $\mathrm{t}(15 ; 17)$ is a reciprocal translocation between chromosomes 15 and 17 that blocks the cell's response to retinoic acid (normal growth factor), the cell remaining in an immature stage, as a promyelocyte (1). There are two main subtypes of APL - hypergranular (typical) and hypogranular (microgranular) variants. Also, there are other different cytogenetic anomalies found in other subtypes of APL with different prognostic implications (5). In less than $5 \%$ of APL cases, the RAR $\alpha$ gene fusions with other genes like PLZF, NPM1, NUMA1, and STAT5B (6). Some of these fusions have a negative impact on the treatment response and, as a consequence, on survival.

The morphological aspect is also characteristic. In the hypergranular variant, large atypical promyelocytes are found, with large, round, or incised nuclei, with visible, large nucleoli. The cytoplasm is abundant and contains a high number of azurophilic granules and Auer rods. In the microgranular variant, with a less favorable prognosis, granules are absent or rare, and nuclei are bilobed; patients also usually present leukocytosis, also an unfavorable prognosis feature (1).

APL is characterized by frequent hemorrhagic complications at debut, which appear with a higher prevalence compared to the other AML subtypes (7). The coagulopathy represents the principal cause of early mortality in APL (8). The most frequent ones are disseminated intravascular coagulation with secondary fibrinolysis and primary fibrinolysis. These are generated by the release of tissue factor from the granule content of the atypical promyelocytes $(7,8)$.

The specific treatment includes ATRA in high doses. It is immediately initiated at the first suspicion of APL, because it overlaps the maturation blockage and induces cell differentiation, thus preventing coagulation complications. ATRA is associated with chemotherapy in protocols such as AIDA, or with arsenic trioxide $(9,10)$, depending on the risk classification. Remission is achieved for a high percentage of patients, $85-90 \%$ being able to obtain complete remission (11). Afterward, the treatment is continued with risk-adapted consolidation treatment and two-years maintenance treatment (10).

Unfavorable prognostic factors described in APL are: increased WBC (over 10,000/ $\mathrm{mm}^{3}$ ), decreased platelet count (under $40,000 / \mathrm{mm}^{3}$ ), male 
gender, presence of CD34, CD56, and the T cell antigen $\mathrm{CD} 2$ on the surface of atypical promyelocytes, presence of FLT3-ITD mutation (12).

Hemorrhage and infection are among the most common causes of induction failure (11). More frequently, infection is encountered in older male patients (13). Even though our patient developed both hemorrhage and infection, she responded to induction therapy and also maintained the response. One of the reasons this happened is the correct treatment applied promptly at the occurrence of complications.

The prognosis of our patient was intermediate. She had APL (a good prognosis subtype of AML) with $\mathrm{t}(15 ; 17)$, which has chemosensitivity. Also, she had a de novo disease, was young and without comorbidities, had a favorable ECOG, and a low WBC (14). However, she presented with a hemorrhagic complication and also with severely decreased platelet count.

The bleeding risk in our patient was very high. The most critical period for APL patients is the time until complete remission when severe bleedings may occur. Afterward, we can talk about healing, taking into account that, except for the severe thrombocytopenia, she had only good prognostic factors.

\section{REFERENCES}

1. Ryan MM. Acute Promyelocytic Leukemia: A Summary. J Adv Pract Oncol. 2018;9(2):178-187.

2. Sticco K, Ahmed T, Salman H. Intracranial acute promyelocytic leukemia at presentation - A case-based discussion. Leuk Res Rep. 2019;11:41-45.

3. Onisai M, Vladareanu AM, Spinu A et al. Idiopathic thrombocytopenic purpura (ITP) - new era for an old disease. Rom J Intern Med. 2019; 57(4):273-283.

4. Onisâi $M$, Vlădăreanu $A M$, Delcea $C$, Ciorăscu M, Bumbea $H$, Nicolescu A, Voican I, Filipescu A, Rotaru O, Vlădăreanu R. Perinatal outcome for pregnancies complicated with thrombocytopenia. J Matern Fetal Neonatal Med. 2012;25(9):1622-6.

5. Akhtar K, Ahmad S, Sherwani RK. Acute promyelocytic leukemia, hypogranular variant: a rare presentation. Clin Pract. 2011;1(1):e11.

6. Mistry AR, Pedersen EW, Solomon E, Grimwade D. The molecular pathogenesis of acute promyelocytic leukaemia: implications for the clinical management of the disease. Blood Rev. 2003;17(2):71-97.

7. Mantha S, Tallman M, Soff G. What's new in the pathogenesis of the coagulopathy in acute promyelocytic leukemia? Curr Opin Hematol. 2016;23(2):121-126.

8. Thomas X. Acute Promyelocytic Leukemia: A History over 60 Years-From the Most Malignant to the Most Curable Form of Acute Leukemia. Oncol Ther. 2019;7(1):33-65.
We would like to underline the very fragile status of our patient. She had severe aplasia, severe leucopenia with increased sepsis risk, severe thrombocytopenia with increased bleeding risk, and also a history of bleeding complications. She is not a typical patient to undergo surgery. The intervention was a risk, but conservative treatment was not successful.

Therefore, taking into account both risks and benefits, we decided in favor for the surgery. Usually, an initial abnormal coagulation profile has a stronger correlation with severe bleeding episodes than initial thrombocytopenia (14), partially explaining the favorable evolution.

\section{CONCLUSIONS}

In the setting of APL with coagulopathy and surgery-requiring infection, it is difficult to decide whether the benefits of surgery will outweigh the risks. Sometimes, it is better to choose a more aggressive approach, assume the risks, and try to prevent possible complications. This case sustains the importance of teamwork in hematologic patients.

Conflict of interest: none declared Financial support: none declared

9. Larson RA. Initial treatment of acute promyelocytic leukemia in adults. UpToDate. 2018. Available at: www.uptodate.com/contents/ initial-treatment-of-acute-promyelocytic-leukemia-in-adults (accessed on June 25, 2018).

10. National Comprehensive Cancer Network. NCCN Practice Guidelines in Oncology: Acute Myeloid Leukemia. V.1.2018. Available at: www. nccn.org/professionals/physician_gls/pdf/aml.pdf (accessed on June 25, 2018)

11. Kotiah S, Besa E, Talavera F, Sacher R. Acute Promyelocytic Leukemia Treatment \& Management. Available at: emedicine. medscape.com/article/1495306-treatment\#d10 (accessed on September 23, 2020).

12. Testa U, Lo-Coco F. Prognostic factors in acute promyelocytic leukemia: strategies to define high-risk patients. Ann Hematol. 2016;95:673-680.

13. de la Serna J, Montesinos P, Vellenga E, et al. Causes and prognostic factors of remission induction failure in patients with acute promyelocytic leukemia treated with all-trans retinoic acid and idarubicin. Blood. 2008;111(7):3395-402.

14. Jang JE, Lee JY, Park HS, et al. Prognostic Factors in Adult Patients with Acute Promyelocytic Leukemia Receiving ATRA-Based Treatment. Blood. 2014;124(21):5274. 